\title{
ON POLYNOMIALS OF SHEFFER TYPE ARISING FROM A CAUCHY PROBLEM
}

\author{
D. G. MEREDITH
}

Received 7 July 2002

\begin{abstract}
A new sequence of eigenfunctions is developed and studied in depth. These theta polynomials are derived from a recent analytic solution of the canonical Cauchy problem for parabolic equations, namely, the inverse heat conduction problem. By appealing to the methods of the operator calculus, it is possible to categorize the new functions as polynomials of binomial and Sheffer types. The connection of the new set with the classical polynomials of Laguerre is carefully examined. Some integral relations involving the Laguerre polynomials and the theta polynomials are presented along with a number of binomial identities. The inverse heat conduction problem is revisited and an analytic solution depending on the generalized theta polynomials is presented.
\end{abstract}

2000 Mathematics Subject Classification: 05A40, 26C05, 35R25.

1. Introduction. In Pettigrew and Meredith [11], an alternative to the classical solution of the inverse heat conduction problem was derived. The classical solution involves derivatives of all orders of the Cauchy data. By overspecifying the problem with the imposition of knowledge of the initial values, and by expanding the Cauchy data in a series of Laguerre polynomials, it was possible to express the solution in a more tractable form for a numerical calculation. The choice of a Laguerre expansion was a logical one for the heat equation and gave rise to an interesting new set of special functions $\Theta_{n}^{ \pm}(x)$. While those functions were closely examined in that paper, their proper place within the realm of special functions was not established.

In this paper, we explore the theta functions in more depth. They derive in a natural way from the coefficients of a series expansion in powers of the Laguerre operator. This pleasing result hints at their intimate connection with the Laguerre polynomials. But it also indicates a fresh approach to the inverse Cauchy problem and suggests a host of new expansions of the operators present in the classical solution of this important inverse problem.

And yet this is only part of the answer to the question of where these special functions should reside. As we will see, the theta functions may be decomposed into a pair of polynomial sequences of binomial type. One set of polynomials is basic for a specific delta operator and the other forms a Sheffer sequence for the first. 
When the theta functions are generalized, a wider class of Sheffer polynomials emerges. This generalized set forms a cross sequence for the basic set in a way completely analogous to the generalized Laguerre polynomials. Indeed, the two generalized sets share a common shift invariant operator.

Key properties of the new polynomials, including a number of integral relations, are derived. Section 6 concludes with a unifying observation: the generalized theta polynomials are the coefficients in a Laguerre series expansion of the fundamental solution of the heat equation. This result is applied in Section 7 to derive an analytic solution of an inverse problem.

2. Operator methods. The classical polynomials of Laguerre, Hermite, Bernoulli, and many others may be neatly classified as polynomials of binomial type. The literature abounds with examples of their use, in the theory of functions, in probability theory and statistics, and of course, in combinatorics. A complete discussion of their abundant properties may be found in the methods of the finite operator calculus. Such developments not only provide a unified approach to the study of each of the classical polynomials, but also admit an effective means of relating two different polynomial sets. Sometimes referred to as the Heaviside calculus, after an early proponent, operational methods have been, over the years, both welcomed for their utility and derided for their lack of rigour. The umbral calculus, as it came to be called, was provided a firm foundation in the works of Rota et al. [10, 13, 14]. Further refinements may be found in Loeb and Rota [9] and Di Bucchianico et al. [4].

In the pages that follow, we will make extensive use of the operational and umbral calculi and, for the sake of completeness, we include a few basic definitions from the work of Rota et al. [13] and Loeb and Rota [9]. All operators, unless otherwise stated, will be assumed to act on the vector space of polynomials of degree less than or equal to $n$. The shift operator is defined by

$$
E^{y} f(x)=f(x+y) .
$$

The operator $M$ is a delta operator if it commutes with the shift operator and if it has the property that $M x=c \neq 0$. A polynomial sequence $p_{n}(x)$ is a sequence of basic polynomials for the delta operator $M$ if $p_{n}(0)=0$ for all $n>0, p_{0}(x)=1$, and $M p_{n}(x)=n p_{n-1}(x)$. Any basic sequence of polynomials is also said to be a sequence of polynomials of binomial type, that is to say, they satisfy the identities

$$
E^{y} p_{n}(x)=\sum_{k=0}^{n}\left(\begin{array}{l}
n \\
k
\end{array}\right) p_{k}(x) p_{n-k}(y) .
$$

A polynomial sequence $q_{n}(x)$ is a Sheffer set for the delta operator $M$ if $q_{0}(x)=c \neq 0$ and $M q_{n}(x)=n q_{n-1}(x)$. The basic set $p_{n}(x)$ and a Sheffer set $q_{n}(x)$ for $M$ are related by a shift-invariant operator $S$ such that $q_{n}(x)=$ $S^{-1} p_{n}(x)$. 
And finally, a cross-sequence $p_{n}^{(\lambda)}(x)$ is a polynomial sequence derived from the application of a one-parameter group $P^{-\lambda}$ of shift-invariant operators to a sequence of polynomials of binomial type.

For example,

$$
Q=\frac{D}{D-I}
$$

is a delta operator whose sequence of basic polynomials is the basic Laguerre set

$$
l_{n}(t)=t(D-I)^{n} t^{n-1}=t e^{t} D^{n} e^{-t} t^{n-1}
$$

A cross-sequence for $l_{n}(t)$ relative to $Q$ is the set of generalized Laguerre polynomials $l_{n}^{\alpha}(t)=(I-D)^{\alpha+1} l_{n}(t)$. For each choice of $\alpha$, the cross-sequence becomes a Sheffer set relative to the operator $(I-D)^{-\alpha-1}$. Note that these Laguerre sets differ from the common usage of the term by a factor of $n !$. In Lebedev [8], for example, the generalized Laguerre polynomials are defined by

$$
L_{n}^{\alpha}(t)=e^{t} \frac{t^{-\alpha}}{n !} \frac{d^{n}}{d t^{n}}\left(e^{-t} t^{n+\alpha}\right)
$$

so that the basic set referred to above is related to the case $\alpha=-1$ :

$$
l_{n}(t)=l_{n}^{-1}(t)=n ! L_{n}^{(-1)}(t) .
$$

3. The theta functions. In Pettigrew and Meredith [11], the following theta functions were derived:

$$
\begin{gathered}
\Theta_{n}^{-}(x)=\sum_{k=0}^{\infty}\left(\begin{array}{c}
n+k-1 \\
n
\end{array}\right) \frac{x^{2 k}}{(2 k) !}, \quad n=0,1,2, \ldots, \\
\Theta_{n}^{+}(x)=\sum_{k=0}^{\infty}\left(\begin{array}{c}
n+k-1 \\
n
\end{array}\right) \frac{x^{2 k+1}}{(2 k+1) !}, \quad n=0,1,2, \ldots
\end{gathered}
$$

They possess the corresponding generating functions:

$$
\begin{gathered}
G^{-}(x, s)=\cosh \left(\frac{x}{\sqrt{1-s}}\right)=\sum_{n=0}^{\infty} \Theta_{n}^{-}(x) s^{n}, \quad|s|<1, \\
G^{+}(x, s)=\sqrt{1-s} \sinh \left(\frac{x}{\sqrt{1-s}}\right)=\sum_{n=0}^{\infty} \Theta_{n}^{+}(x) s^{n}, \quad|s|<1 .
\end{gathered}
$$

The following theorem is required [9]. 
THEOREM 3.1. Any shift-invariant operator $T$ may be expanded as

$$
T=\sum_{n=0}^{\infty} \frac{c_{n}}{n !} Q^{n}
$$

for some delta operator $Q$ and its basic set $p_{n}(t)$, where $c_{n}=\left[T p_{n}(t)\right]_{t=0}$. (Of course, the sum is finite when applied to polynomials.)

Now the theta functions $\Theta_{n}^{ \pm}(x)$ are closely connected with the Laguerre polynomials in the following way.

Proposition 3.2. The theta functions $\Theta_{n}^{ \pm}(x)$ are the coefficients in the expansion in powers of $Q=D /(D-I)$ of the shift-invariant operators $\cosh (x \sqrt{I-D})$ and $\sinh (x \sqrt{I-D}) / \sqrt{I-D}$.

Proof. The result follows at once from (3.2) upon writing

$$
\begin{aligned}
& G^{-}\left(x, \frac{D}{D-I}\right)=\cosh (x \sqrt{I-D}), \\
& G^{+}\left(x, \frac{D}{D-I}\right)=\sqrt{I-\frac{D}{D-I}} \sinh (x \sqrt{I-D}) .
\end{aligned}
$$

Alternatively, we could take as our starting point Theorem 3.1 and use the umbral calculus to derive the theta functions $\Theta^{ \pm}(x)$ from scratch. For example, consider the shift-invariant operator $T=\cosh (x \sqrt{I-D})$ and the delta operator $Q=D /(D-I)$ of the Laguerre polynomials $l_{n}(t)$. Then Theorem 3.1 yields

$$
\cosh (x \sqrt{I-D})=\sum_{n=0}^{\infty} \frac{c_{n}(x)}{n !}\left(\frac{D}{D-I}\right)^{n},
$$

where

$$
\begin{aligned}
c_{n}(x) & =\left[\cosh (x \sqrt{I-D}) l_{n}(t)\right]_{t=0} \\
& =\left[\sum_{k=0}^{\infty} \frac{x^{2 k}}{(2 k) !}(I-D)^{k} l_{n}(t)\right]_{t=0} \\
& =n ! \sum_{k=0}^{\infty} \frac{x^{2 k}}{(2 k) !}\left(\begin{array}{c}
n+k-1 \\
n
\end{array}\right) \\
& =n ! \Theta_{n}^{-}(x) .
\end{aligned}
$$

In a like manner, we find that $\Theta_{n}^{+}(x)$ as defined in (3.1) emerge as coefficients in the operator expansion

$$
\frac{\sinh (x \sqrt{I-D})}{\sqrt{I-D}}=\sum_{n=0}^{\infty} \Theta_{n}^{+}(x)\left(\frac{D}{D-I}\right)^{n} .
$$


4. Polynomials of binomial type. We have Theorems 4.1 and 4.3 and Lemma 4.2 from Rota et al. [13].

THEOREM 4.1. Let $M$ be a delta operator. The ring of formal power series is isomorphic to the ring of shift-invariant operators so that $\sum_{n=0}^{\infty}\left(c_{n} / n\right.$ !) $t^{n}$ is carried into $\sum_{n=0}^{\infty}\left(c_{n} / n !\right) M^{n}$.

LEMMA 4.2. A shift-invariant operator $M$ is a delta operator if and only if its corresponding formal power series $m(t)$ has the properties $m(0)=0$ and $m^{\prime}(0) \neq 0$.

THEOREM 4.3. Let $M$ be a delta operator with basic set $p_{n}(x)$ and let $S$ be a shift-invariant operator for the corresponding Sheffer set $q_{n}(x)$. Let $s(t)$ and $m(t)$ be the formal power series associated with $S$ and $M$, respectively, where $m^{-1}(t)$ denotes the formal power series inverse to $m(t)$. Then the generating function for the sequence $q_{n}(x)$ is given by

$$
\frac{e^{x m^{-1}(t)}}{s\left(m^{-1}(t)\right)}=\sum_{n=0}^{\infty} \frac{q_{n}(x)}{n !} t^{n}
$$

We are thus in a position to derive our main result for this section as follows.

THEOREM 4.4. The theta functions $\Theta_{n}^{-}(x)$ have the decomposition

$$
\Theta_{n}^{-}(x)=\left[\frac{e^{x}}{2} \frac{p_{n}(-x)}{n !}+\frac{e^{-x}}{2} \frac{p_{n}(x)}{n !}\right]
$$

where the $p_{n}(x)$ are polynomials of degree $n$, basic for the delta operator

$$
M(D)=\frac{D^{2}-2 D}{(D-I)^{2}}
$$

Proof. We have

$$
\sum_{n=0}^{\infty} \Theta_{n}^{-}(x) t^{n}=\cosh \left(\frac{x}{\sqrt{1-t}}\right)=\frac{1}{2}\left(e^{x} e^{-x(1-1 / \sqrt{1-t})}+e^{-x} e^{x(1-1 / \sqrt{1-t})}\right) .
$$

Writing $m^{-1}(t)=(1-1 / \sqrt{1-t}), s(t)=1$, we have

$$
m(t)=\frac{t^{2}-2 t}{(t-1)^{2}}
$$

Now, $m(0)=0$ and $m^{\prime}(0) \neq 0$. By Lemma 4.2, $m(t)$ has a formal power series that corresponds, under the isomorphism of Theorem 4.1, to the delta operator $M(D)=\left(D^{2}-2 D\right) /(D-I)^{2}$ if $t$ corresponds to the delta operator $D$. Thus, by Theorem 4.3, there exists a sequence of polynomials $p_{n}(x)$, which are basic 
for $M(D)$, having the generating function

$$
e^{ \pm x(1-1 / \sqrt{1-t})}=\sum_{n=0}^{\infty} \frac{p_{n}( \pm x)}{n !} t^{n} .
$$

Equation (4.2) follows upon comparing (4.6) and (4.4).

An immediate consequence is the identification of $p_{n}(x)$ as a sequence of polynomials of binomial type for the operator $M$. That is, the defining characteristic of these polynomials is (2.2). An explicit form for the new polynomials will be provided in Section 5. It will suffice for the moment to give the form of the first few polynomials:

$$
p_{o}(x)=1, \quad p_{1}(x)=-\frac{x}{2}, \quad p_{2}(x)=-\frac{3}{4} x+\frac{1}{4} x^{2} .
$$

Now turning to an examination of the functions $\Theta_{n}^{+}(x)$, we write

$$
\Theta_{n}^{+}(x)=\frac{e^{x}}{2} \frac{q_{n}(-x)}{n !}-\frac{e^{-x}}{2} \frac{q_{n}(x)}{n !}
$$

for some polynomials $q_{n}(x)$. Now, the $q_{n}(x)$ do not form a basic set, but rather form a Sheffer set. That is,

$$
\Theta_{n}^{-}(x)=D \Theta_{n}^{+}(x)=D\left[\frac{e^{x}}{2} \frac{q_{n}(-x)}{n !}-\frac{e^{-x}}{2} \frac{q_{n}(x)}{n !}\right]
$$

so that

$$
p_{n}(x)=S q_{n}(x) \quad \text { where } S=I-D .
$$

Thus, $q_{n}(x)$ is a Sheffer set relative to the operator $M(D)=\left(D^{2}-2 D\right) /(D-I)^{2}$ and basic polynomials $p_{n}(x)$. We immediately have the binomial relation

$$
q_{n}(x+y)=\sum_{k \geq 0}\left(\begin{array}{l}
n \\
k
\end{array}\right) q_{k}(x) p_{n-k}(y) .
$$

The first few Sheffer polynomials are

$$
q_{0}(x)=1, \quad q_{1}(x)=-\frac{1}{2}-\frac{1}{2} x, \quad q_{2}(x)=-\frac{1}{4}-\frac{1}{4} x+\frac{1}{4} x^{2} .
$$

In the following sections, the functions $p_{n}$ and $q_{n}$ and their subsequent generalizations will be simply referred to as theta polynomials. An extensive review of the literature on binomial type polynomials and their related Sheffer sets is provided in [13]. However, no mention is given to functions which are the product of exponentials and Sheffer polynomials, the class to which our theta functions belong. Yet there are a number of exceptional special functions that belong to this category. We draw the reader's attention to the Laguerre 
functions and the Sturmian functions. The former are a natural set of orthogonal functions based on the Laguerre polynomials and the reader is directed to the work of Lanczos [7], for example. The Sturmian functions are also closely linked to the Laguerre polynomials and have seen extensive application in recent years. Rotenberg first coined the term Sturmians as the functions in question are the solutions to a Sturm-Liouville type equation (see [15]). Sturmians are most notable for their application to atomic scattering problems, owing to their obvious relationship to solutions of the hydrogenic equation. The reader is directed to Avery [1] for an overview of the theory and application of these remarkable special functions.

Both the Laguerre functions and Sturmians represent complete orthogonal sets with discrete eigenvalues. In this regard, the theta functions and polynomials of the present work do not possess the usual integral orthogonality relations since they represent solutions to a third-order differential equation, as we will see in Section 5.

5. Generalized theta polynomials. The binomial coefficient $\left(\begin{array}{c}n+k-1 \\ n\end{array}\right)$ appearing in each of the expressions for $\Theta_{n}^{ \pm}(x)$ has an interesting combinatorial interpretation. It enumerates the number of free distributions of $n$ identical elements to $k$ different labels. (In our case, the reader will recall the coefficient derives from the zero condition of the Laguerre polynomials $L_{n}^{(k-1)}(0)$.) It is of considerable interest to examine the impact of the more general binomial coefficient

$$
\left(\begin{array}{c}
n+k-\alpha \\
n
\end{array}\right)
$$

on the theory of the theta functions already considered. If $\alpha$ is an integer, then the combinatorial interpretation represents an adjustment to the number of distinguishable labels. For arbitrary $\alpha$, it may be considered to derive from the zero condition of the Laguerre polynomials $L_{n}^{(k-\alpha)}(0)$, in which case we may define a generalized set of theta functions as follows:

$$
\begin{aligned}
& \Theta_{n}^{-}(x, \alpha)=\sum_{k \geq 0}\left(\begin{array}{c}
n+k-\alpha \\
n
\end{array}\right) \frac{x^{2 k}}{(2 k) !}, \\
& \Theta_{n}^{+}(x, \alpha)=\sum_{k \geq 0}\left(\begin{array}{c}
n+k-\alpha \\
n
\end{array}\right) \frac{x^{2 k+1}}{(2 k+1) !} .
\end{aligned}
$$

The corresponding generating functions are

$$
\begin{gathered}
(1-s)^{\alpha-1} \cosh \frac{x}{\sqrt{1-s}}=\sum_{n=0}^{\infty} \Theta_{n}^{-}(x, \alpha) s^{n}, \\
(1-s)^{\alpha-1 / 2} \sinh \frac{x}{\sqrt{1-s}}=\sum_{n=0}^{\infty} \Theta_{n}^{+}(x, \alpha) s^{n} .
\end{gathered}
$$


That is, the generalized functions remain the coefficients in the expansion in powers of $Q=D /(D-I)$ of certain shift-invariant operators. In particular, $\Theta_{n}^{ \pm}(x, \alpha)$ derive from the operator expansions

$$
\begin{aligned}
(I-D)^{1-\alpha} \cosh (x \sqrt{I-D}) & =\sum_{n=0}^{\infty} \Theta_{n}^{-}(x, \alpha)\left(\frac{D}{D-I}\right)^{n}, \\
(I-D)^{1 / 2-\alpha} \sinh (x \sqrt{I-D}) & =\sum_{n=0}^{\infty} \Theta_{n}^{+}(x, \alpha)\left(\frac{D}{D-I}\right)^{n} .
\end{aligned}
$$

Now, as for the polynomials that comprise the generalized theta functions, we proceed as follows. We define

$$
\Delta_{n}^{ \pm}(x, \alpha)=\Theta_{n}^{-}\left(x, \frac{\alpha}{2}+1\right) \pm \Theta_{n}^{+}\left(x, \frac{\alpha}{2}+\frac{1}{2}\right) .
$$

In particular, we may express

$$
\Delta_{n}^{-}(x, \alpha)=e^{-x} \frac{p_{n}^{(\alpha)}(x)}{n !}
$$

for some polynomials $p_{n}^{(\alpha)}(x)$. The power series for $\Delta_{n}^{-}$can be determined as follows:

$$
\begin{aligned}
\Delta_{n}^{-}(x, \alpha) & =\Theta_{n}^{-}\left(x, \frac{\alpha}{2}+1\right)-\Theta_{n}^{+}\left(x, \frac{\alpha}{2}+\frac{1}{2}\right) \\
& =\sum_{k \text { even }}\left(\begin{array}{c}
n+\frac{k-\alpha}{2}-1 \\
n
\end{array}\right) \frac{x^{k}}{k !}-\sum_{k \text { odd }}\left(n+\frac{k-\alpha}{2}-1\right) \frac{x^{k}}{k !} \\
& =\sum_{k=0}^{\infty}\left(n+\frac{k-\alpha}{2}-1\right) \frac{(-x)^{k}}{k !} .
\end{aligned}
$$

The binomial coefficient appearing in (5.7) will be examined in more detail in the next section.

Proposition 5.1. The functions $p_{n}^{(\alpha)}(x)$ represent a cross-sequence associated with the basic set $p_{n}(x)$ and $p_{n}^{(\alpha)}(x)=(I-D)^{-\alpha} p_{n}(x)$.

Proof. From (5.3), the generating function for $\Delta_{n}^{-}(x, \alpha)$ is

$$
(1-s)^{\alpha / 2} e^{-x / \sqrt{1-s}}=\sum_{n=0}^{\infty} \Delta_{n}^{-}(x, \alpha) s^{n}=e^{-x} \sum_{n=0}^{\infty} \frac{p_{n}^{(\alpha)}(x)}{n !} s^{n} .
$$

That is,

$$
\frac{1}{(1-t)^{\alpha}} e^{x t}=\sum_{n=0}^{\infty} \frac{p_{n}^{(\alpha)}(x)}{n !}(m(t))^{n}, \quad m(t)=\frac{t^{2}-2 t}{(t-1)^{2}}
$$


Under the isomorphism of Theorem 4.1,

$$
\left(I-D_{t}\right)^{-\alpha} e^{x D_{t}}=\sum_{n=0}^{\infty} \frac{p_{n}^{(\alpha)}(x)}{n !}\left(M\left(D_{t}\right)\right)^{n}
$$

and by Theorem 3.1,

$$
p_{n}^{(\alpha)}(x)=\left[\left(I-D_{t}\right)^{-\alpha} e^{x D_{t}} p_{n}(t)\right]_{t=0}=(I-D)^{-\alpha} p_{n}(x) .
$$

For each choice of $\alpha$, a particular Sheffer set of the basic polynomials is generated. The case $\alpha=1$ reproduces the Sheffer set $q_{n}(x)$ of an earlier section. The case $\alpha=-1$ generates a Sheffer set closely linked to the fundamental solution of the heat conduction equation, as we will see shortly.

It is worth noting at this stage the remarkable similarities shared by the cross-sequences of the new polynomials and the familiar Laguerre polynomials. Note that $p_{n}^{(\alpha)}(x)=(I-D)^{-\alpha} p_{n}(x)=S^{-1} p_{n}(x)$ and recall that $l_{n}^{-(\alpha+1)}(x)=$ $(I-D)^{-\alpha} l_{n}(x)=S^{-1} l_{n}(x)$. In other words, the generalized theta polynomials $p_{n}^{(\alpha)}(x)$ and the associated Laguerre polynomials $l_{n}^{-(\alpha+1)}(x)$ share the same invertible operator $S=(I-D)^{\alpha}$, but applied to a different basic set in each case.

We explore these new polynomials in more depth. To this point, then, we have that $p_{n}(x)$ is a basic set of polynomials of binomial type relative to the delta operator $M=\left(D^{2}-2 D\right) /(D-I)^{2}$ and therefore satisfies the identity

$$
p_{n}(x+y)=\sum_{k=0}^{n}\left(\begin{array}{l}
n \\
k
\end{array}\right) p_{k}(x) p_{n-k}(y) .
$$

The generalized set $p_{n}^{(\alpha)}(x)$ represents a Sheffer sequence for each fixed $\alpha$ relative to the basic set $p_{n}(x)$ and the invertible operator $S=(I-D)^{\alpha}$. Applying $\left(I-D_{x}\right)^{-\alpha}\left(I-D_{y}\right)^{-\beta}$ to each side of (5.12), we obtain the interesting binomial identity

$$
p_{n}^{(\alpha+\beta)}(x+y)=\sum_{k=0}^{n}\left(\begin{array}{l}
n \\
k
\end{array}\right) p_{k}^{(\alpha)}(x) p_{n-k}^{(\beta)}(y) .
$$

As already mentioned, the apparatus of the operational calculus effectively permits the expression of one polynomial sequence in terms of another. If we examine the operator $M$ more closely, we observe the following:

$$
M(D)=\frac{D^{2}-2 D}{\left(D^{2}-2 D\right)+I}=\frac{A}{A+I},
$$

where $A=D^{2}-2 D$ and, recalling the Laguerre operator $Q=D /(D-I)$, we find that $M=Q(-A)$, which expresses the delta operator $M$ as the umbral composition (to use the language of [13]) of the Laguerre operator $Q$ and another delta 
operator $A$. It is noteworthy that the binomial properties of both these operators have been extensively studied in the literature. In the case of the operator $-A / 2$, the corresponding polynomial set has been investigated by Carlitz [2].

THE DifFERENTIAL EQUATION. It is also possible to derive the connection between the theta polynomials and the set $x^{n}$, which is basic for the delta operator $D$. It is a simple matter to show that

$$
x^{n}=W_{n, \alpha} p_{n}^{(\alpha)}(x), \quad \text { where } W_{n, \alpha}=\left(M^{\prime}\right)^{-1} P^{n+1} S,
$$

with $M=D P$ and $M^{\prime}$ denoting differentiation with respect to $D$. Then,

$$
W_{n, \alpha}=\frac{(-1)^{\alpha}}{2} \frac{(D-2 I)^{n+1}}{(D-I)^{2 n-\alpha-1}} .
$$

This connection can be used to derive the differential equation and its associated eigenvalue problem. Rota et al. [13] take a different approach and establish the following general result.

THEOREM 5.2. For any Sheffer sequence $s_{n}(x)$ with delta operator $Q$ and operator $S$, there exists an essentially selfadjoint operator $A$ having integer eigenvalues $n=0,1,2, \ldots$ for the eigenfunctions $s_{n}(x)$, where

$$
A=\sum_{k \geq 1} \frac{a_{k}+x b_{k}}{k !} R^{k}
$$

with

$$
\begin{aligned}
& a_{k}=-\left[(\log S)^{\prime} Q\left(Q^{\prime}\right)^{-1} r_{k}(x)\right]_{x=0}, \\
& b_{k}=\left[Q\left(Q^{\prime}\right)^{-1} r_{k}(x)\right]_{x=0} .
\end{aligned}
$$

The operator $R$ is any delta operator possessing basic set $r_{k}(x)$.

If we choose $R=D$ with basic set $x^{k}$, it is a simple matter to establish the following theorem.

THEOREM 5.3. The Sheffer set $p_{n}^{(\alpha)}(x)$, relative to the delta operator $M(D)=$ $\left(D^{2}-2 D\right) /(D-I)^{2}$ and shift-invariant operator $S=(I-D)^{\alpha}$, represents a sequence of eigenfunctions for the operator

$$
L(D)=\frac{x}{2} D^{3}-\left(\frac{\alpha+3 x}{2}\right) D^{2}+(\alpha+x) D
$$

with eigenvalues $n=0,1,2, \ldots$

At this point, we observe a significant departure from the usual secondorder ordinary differential equations satisfied by the classical sets of orthogonal polynomials, including the Laguerre polynomials. Nevertheless, the case 
remains that the new polynomial set forms a complete sequence of eigenfunctions with integer eigenvalues $n$, as is the situation with the classical polynomials, albeit this time relative to an essentially selfadjoint third-order differential equation.

A study of this differential equation would provide an alternative route to the development of the new polynomials. Such an investigation could prove to be as rich as the current approach. After all, third-order ordinary differential equations have received scant attention over the years, especially in comparison to equations of first, second, and fourth order. Such is the dearth that it is possible to find concise, yet comprehensive surveys of the literature on third-order equations (see, e.g., [6, 16]).

We conclude this section with a derivation of the recurrence formula required for generating the theta polynomials, which will be needed in Section 7 . From (5.8), the generating function for the theta polynomials satisfies the differential equation

$$
g_{s s}+\frac{(\alpha-3 / 2)}{(1-s)} g_{s}+\left[\frac{\alpha(\alpha-1)}{4(1-s)^{2}}-\frac{x^{2}}{4(1-s)^{3}}\right] \mathfrak{g}=0 .
$$

Substituting (5.8) into (5.20) and collecting like powers of $s$, we find

$$
\begin{gathered}
p_{n+3}^{(\alpha)}+\alpha_{n} p_{n+2}^{(\alpha)}+\beta_{n} p_{n+1}^{(\alpha)}+\gamma_{n} p_{n}^{(\alpha)}=0, \\
\alpha_{n}=\left(\alpha-\frac{3}{2}\right)-3(n+1), \\
\beta_{n}=3 n(n+1)-2(n+1)\left(\alpha-\frac{3}{2}\right)+\frac{\alpha(\alpha-1)-x^{2}}{4}, \\
\gamma_{n}=-(n-1) n(n+1)+n(n+1)\left(\alpha-\frac{3}{2}\right)-\frac{\alpha(\alpha-1)(n+1)}{4}, \\
p_{0}^{(\alpha)}(x)=1, \quad p_{1}^{(\alpha)}(x)=-\frac{(\alpha+x)}{2}, \\
p_{2}^{(\alpha)}(x)=\frac{\alpha(\alpha-2)}{4}+\frac{(2 \alpha-3) x}{4}+\frac{x^{2}}{4} .
\end{gathered}
$$

Alternatively, an explicit expression for the polynomials follows from a rearrangement of the power series (5.7):

$$
\frac{p_{n}^{(\alpha)}(x)}{n !}=\sum_{m=0}^{n}\left(\sum_{k=0}^{m}\left(\begin{array}{c}
m \\
k
\end{array}\right)\left(\begin{array}{c}
n+\frac{k-\alpha}{2}-1 \\
n
\end{array}\right)(-1)^{k}\right) \frac{x^{m}}{m !} .
$$

6. Integral relations. In this section, we employ the operational calculus to derive a number of new integral relations involving Laguerre polynomials. Some binomial identities stemming from the foregoing theory are also presented. We conclude with an intriguing result that ties the theory of the theta polynomials to the fundamental solution of the heat equation. 
It is often convenient to express a differential operator in integral form. In fact, Di Bucchianico [3] has shown that every linear shift-invariant operator has a corresponding integral representation with respect to a complex Borel measure on the real line. We will derive the following result which generalizes a class of operators found in the literature.

THEOREM 6.1. Let $a, b \in \mathbb{R}$ with $b>0$. Then,

$$
\frac{(D-a I)^{n}}{(D-b I)^{n+v}} \phi(x)=\frac{(-1)^{v} n !}{\Gamma(n+v)} \int_{0}^{\infty} t^{v-1} e^{-b t} L_{n}^{(v-1)}((b-a) t) \phi(x+t) d t,
$$

where $v>0$ and $n=0,1,2, \ldots$

Proof. Denote the integral operator by $K$. Then $K$ is clearly shift invariant since

$$
\begin{aligned}
K E^{y} \phi(x) & =\frac{(-1)^{v} n !}{\Gamma(n+v)} \int_{0}^{\infty} t^{v-1} e^{-b t} L_{n}^{(v-1)}((b-a) t) \phi(x+y+t) d t \\
& =E^{y} K \phi(x) .
\end{aligned}
$$

Therefore, we may expand, by Theorem 3.1,

$$
K=\sum_{m=0}^{\infty} \frac{a_{m}}{m !} D^{m},
$$

where

$$
\begin{aligned}
\frac{a_{m}}{m !} & =\frac{1}{m !}\left[K x^{m}\right]_{x=0} \\
& =\frac{(-1)^{v} n !}{m ! \Gamma(n+v)} \int_{0}^{\infty} t^{m+v-1} e^{-b t} L_{n}^{(v-1)}((b-a) t) d t \\
& =\frac{(-1)^{v} n !}{b^{m+v} m ! \Gamma(n+v)} \int_{0}^{\infty} \tau^{m+v-1} e^{-\tau} L_{n}^{(v-1)}(\xi \tau) d \tau, \quad \text { where } \xi=\frac{b-a}{b} \\
& =\frac{(-1)^{v} n !}{b^{m+v} m ! \Gamma(n+v)} \int_{0}^{\infty} \tau^{m+v-1} e^{-\tau} \sum_{k=0}^{n} \frac{\Gamma(n+v)}{\Gamma(k+v)} \frac{(-1)^{k} \xi^{k} \tau^{k}}{k !(n-k) !} d \tau \\
& =\sum_{k=0}^{n} \frac{(-1)^{k+v}}{b^{m+v}}\left(\begin{array}{c}
m+k+v-1 \\
m
\end{array}\right)\left(\begin{array}{c}
n \\
k
\end{array}\right) \xi^{k} .
\end{aligned}
$$

Therefore,

$$
\begin{aligned}
\sum_{m=0}^{\infty} \frac{a_{m}}{m !} s^{m} & =\sum_{m=0}^{\infty} \sum_{k=0}^{n}(-1)^{k+v} \frac{(b-a)^{k}}{b^{m+v+k}}\left(\begin{array}{l}
n \\
k
\end{array}\right)\left(\begin{array}{c}
m+v+k-1 \\
m
\end{array}\right) s^{m} \\
& =\sum_{k=0}^{n}\left(\begin{array}{l}
n \\
k
\end{array}\right)(b-a)^{k} \frac{(-1)^{k+v}}{b^{k+v}} \sum_{m=0}^{\infty}\left(\begin{array}{c}
m+v+k-1 \\
m
\end{array}\right)\left(\frac{s}{b}\right)^{m} \\
& =\sum_{k=0}^{n}\left(\begin{array}{l}
n \\
k
\end{array}\right)\left(\frac{b-a}{s-b}\right)^{k}\left(\frac{1}{s-b}\right)^{v}=\frac{(s-a)^{n}}{(s-b)^{n+v}}
\end{aligned}
$$


where the interchange of summations in (6.4) is valid as the infinite sum is absolutely convergent for all $s$. The conclusion of the proof follows once again from an application of the isomorphism Theorem 4.1.

In a form reminiscent (yet again) of the familiar Laguerre operator

$$
\frac{D}{D-I} \phi(x)=-\int_{0}^{\infty} e^{-t} \phi^{\prime}(x+t) d t
$$

we have the following corollary.

COROLLARY 6.2. The operators $M$ and $P^{n}$ take the integral forms

$$
\begin{aligned}
& M \phi(x)=\frac{D(D-2 I)}{(D-I)^{2}} \phi(x)=-\int_{0}^{\infty} e^{-t} L_{1}(-t) \phi^{\prime}(x+t) d t \\
& P^{n} \phi(x)=\frac{(D-2 I)^{n}}{(D-I)^{2 n}} \phi(x)=\frac{(-1)^{n} n !}{\Gamma(2 n)} \int_{0}^{\infty} t^{n-1} e^{-t} L_{n}^{(n-1)}(-t) \phi(x+t) d t .
\end{aligned}
$$

The polynomials $p_{n}^{(\alpha)}(x)$ can be expressed in integral form using the above integral operator $K$. First, recall (5.16) and the relation

$$
x^{n}=(-1)^{n} \frac{I}{(I-D)^{\beta+n}} l_{n}^{(\beta)}(x), \quad \text { where } l_{n}^{(\beta)}(x)=n ! L_{n}^{(\beta)}(x) .
$$

Then, using our result (6.1), we have

$$
\begin{aligned}
p_{n}^{(\alpha)}(x) & =\frac{2 n !}{(-1)^{\alpha+\beta}} \frac{(D-I)^{n-(\alpha+\beta+1)}}{(D-2 I)^{n+1}} L_{n}^{(\beta)}(x) \\
& =\frac{2(n-\mu) !}{(-1)^{\mu}} \int_{0}^{\infty} t^{\mu} e^{-2 t} L_{n-\mu}^{(\mu)}(t) L_{n}^{(\beta)}(x+t) d t,
\end{aligned}
$$

where $\mu=\alpha+\beta+1$ is an integer such that $\mu \leq n, n=0,1,2, \ldots$ In particular, the special case of $\alpha=-1$ and $\beta=0$ yields the remarkably simple integral relation in the following corollary.

COROLLARY 6.3. The theta polynomial of order $\alpha=-1$ obeys the integral relation

$$
\frac{p_{n}^{(-1)}(x)}{n !}=2 \int_{0}^{\infty} e^{-2 t} L_{n}(t) L_{n}(x+t) d t, \quad n \geq 0 .
$$

We would be remiss if we did not comment on the binomial coefficient appearing in (5.7). In this regard, the integral relation (6.8) permits us to expose yet another integral relation and an associated binomial identity. We begin by noting that

$$
\frac{p_{n}^{(\alpha)}(0)}{n !}=\left(\begin{array}{c}
n-\frac{\alpha}{2}-1 \\
n
\end{array}\right)
$$

as follows from (5.7). Setting $\mu=0$ and $x=0$ in (6.8) and replacing $\alpha$ by $\alpha-k$, we have the following result for the binomial coefficient of (5.7). 
COROLLARY 6.4. The binomial coefficient of (5.7) obeys the integral relation

$$
2 \int_{0}^{\infty} e^{-2 t} L_{n}(t) L_{n}^{(k-\alpha-1)}(t) d t=\left(\begin{array}{c}
n+\frac{k-\alpha}{2}-1 \\
n
\end{array}\right),
$$

where $k$ is a nonnegative integer.

If we expand

$$
L_{n}^{(k-\alpha-1)}(t)=\sum_{m=0}^{n} \frac{\Gamma(k+m-\alpha-1)}{\Gamma(k-\alpha-1) m !} L_{n-m}(t),
$$

substitute into (6.11), and integrate, we find the following result.

COROLLARY 6.5. The binomial coefficient of (5.7) satisfies the identity

$$
\sum_{m=0}^{n}\left(\begin{array}{c}
m+k-\alpha-2 \\
m
\end{array}\right)\left(\begin{array}{c}
2 n-m \\
n
\end{array}\right) \frac{1}{2^{2 n-m}}=\left(\begin{array}{c}
n+\frac{k-\alpha}{2}-1 \\
n
\end{array}\right),
$$

where again $k$ and $n$ are nonnegative integers.

The binomial identity (6.13), expressed in a slightly altered form, is listed in the compendium by Gould [5, Table 3, Identity \#3.53].

Finally, here we take the opportunity to express the connection between the new polynomials for the special case $\alpha=-1$ and the basic Laguerre set $L_{n}^{(-1)}(x)$. From (6.8), it is a simple matter to verify the following corollary.

COROLLARY 6.6. The connection formula relating the theta polynomial of order $\alpha=-1$ to the Laguerre polynomial of order $\alpha=-1$ is given by

$$
\frac{p_{n}^{(-1)}(x)}{n !}=\sum_{k=0}^{n}\left(\begin{array}{c}
n+k \\
n
\end{array}\right) \frac{1}{2^{n+k}} L_{n-k}^{(-1)}(x) .
$$

MORE INTEgRAL RELATIONS AND THE FundAMENTAL SOLUTION OF THE HeAt EquAtion. Returning now to the generating function of the Sheffer polynomials $p_{n}^{(\alpha)}(x)$, we derive some important integral representations. Recall that

$$
(1-s)^{\alpha / 2} e^{-x / \sqrt{1-s}}=e^{-x} \sum_{n=0}^{\infty} \frac{p_{n}^{(\alpha)}(x)}{n !} s^{n},
$$

which converges uniformly and absolutely for each $x \geq 0$ and $|s|<1$. Under the bilinear mapping $u=1 /(1-s)$, the interior of the unit circle transforms conformally to the right half-plane $\operatorname{Re}(u)>1 / 2$, so that the transformed series

$$
(u)^{-\alpha / 2-1} e^{-x(\sqrt{u}-1)}=\sum_{n=0}^{\infty} \frac{p_{n}^{(\alpha)}(x)}{n !} \frac{1}{u}\left(\frac{u-1}{u}\right)^{n}
$$


converges uniformly and absolutely in the half-plane $\operatorname{Re}(u)>1 / 2$. If we treat $u$ as the variable in a Laplace transform, then the left-hand side has an inverse and the right-hand side may be inverted term by term to yield a convergent Laguerre series. (See, e.g., [17].) We have

$$
\frac{e^{-x^{2} / 8 t} D_{-(\alpha+1)}(x / \sqrt{2 t})}{\sqrt{\pi / 2}(2 t)^{-\alpha / 2}}=\sum_{n=0}^{\infty} e^{-x} \frac{p_{n}^{(\alpha)}(x)}{n !} L_{n}(t),
$$

where $D_{-(\alpha+1)}(x / \sqrt{2 t})$ is a parabolic cylinder function. (See [12].)

Thus the Sheffer polynomials $p_{n}^{(\alpha)}(x)$ appear as coefficients in the expansion in Laguerre polynomials of parabolic cylinder functions and, as such, may be expressed in the following integral form.

Proposition 6.7. The theta polynomial of order $\alpha$ obeys the integral relation

$$
p_{n}^{(\alpha)}(x)=n ! e^{x} \int_{0}^{\infty} e^{-t} e^{-x^{2} / 8 t} \frac{D_{-(\alpha+1)}(x / \sqrt{2 t})}{2^{-((\alpha+1) / 2)} \sqrt{\pi} t^{-\alpha / 2}} L_{n}(t) d t
$$

The case $\alpha=-1$ is of particular interest, as the polynomials $p_{n}^{(-1)}(x)$ become coefficients in the Laguerre expansion of the fundamental solution of the heat equation, which after all was our starting point. Indeed, as

$$
D_{0}\left(\frac{x}{\sqrt{2 t}}\right)=e^{-x^{2} / 8 t}
$$

we have

$$
\frac{e^{-x^{2} / 4 t}}{\sqrt{\pi t}}=\sum_{n=0}^{\infty} e^{-x} \frac{p_{n}^{(-1)}(x)}{n !} L_{n}(t), \quad x \geq 0, t>0,
$$

and, in integral form,

$$
p_{n}^{(-1)}(x)=n ! e^{x} \int_{0}^{\infty} \frac{e^{-t-x^{2} / 4 t}}{\sqrt{\pi t}} L_{n}(t) d t
$$

This last relation expresses the new polynomials as an integral involving the Laguerre functions and whose kernel is the source solution of the heat equation.

7. The inverse heat equation revisited. The canonical Cauchy problem for parabolic equations is the inverse heat conduction problem:

$$
u_{x x}=u_{t}, \quad u(0, t)=\phi(t), \quad u_{x}(0, t)=\psi(t)
$$


The classical solution is given as

$$
\begin{aligned}
u(x, t) & =\cosh (x \sqrt{D}) \phi(t)+\frac{\sinh (x \sqrt{D})}{\sqrt{D}} \psi(t) \\
& =\sum_{n=0}^{\infty} \frac{x^{2 n}}{(2 n) !} \phi^{(n)}(t)+\sum_{n=0}^{\infty} \frac{x^{2 n+1}}{(2 n+1) !} \psi^{(n)}(t) .
\end{aligned}
$$

The problem is severely ill posed in that the solution may not depend continuously on the data. This is usually dealt with by some form of regularization, a process that relegates the Cauchy data to a well-posedness class of functions, but which nevertheless leaves an infinite series of derivatives of the mollified data. In Pettigrew and Meredith [11], the authors overspecified the problem with the imposition of the initial condition $u(x, 0)=f(x)$. In this way, a series solution that did not require derivatives of the Cauchy data was derived.

In this regard, the operator methods of the current paper may be used to express the classical solution in a variety of formats, each more suitable for practical calculation than an infinite series of derivatives.

In this section, we consider two applications of the foregoing theory. In the first, we present an operator expansion alternative to that of the classical solution (7.2) and in the second, we construct an analytic solution to an inverse problem using the theta polynomials.

Application 1. An interesting alternative to the classical expansion (7.2) is the following:

$$
\cosh x \sqrt{D} \phi(t)=\sum_{n=0}^{\infty} \frac{B_{n}(x)}{n !} \Delta^{n} \phi(t),
$$

where

$$
B_{n}(x)=\left[\cosh x \sqrt{D}(t)_{n}\right]_{t=0}=\sum_{k=0}^{n} \frac{x^{2 k}}{(2 k) !} s(n, k) k !
$$

with a similar representation for the hyperbolic sine function. The $s(n, k)$ are the Stirling numbers of the first kind. While the substitution of differences for derivatives is not new, it does appear to have been overlooked in application to such Cauchy problems. The class of functions for which the given series converges must be supplied, but we do not pursue this matter here as the polynomials $B_{n}(x)$ are not basic for a delta operator since the corresponding operator representation fails to possess a formal inverse power series.

Application 2. Consider the following one-dimensional heat conduction problem:

$$
\begin{gathered}
u_{x x}=u_{t}, \quad x>0, t>0, \quad-u_{x}(0, t)=\psi(t), \quad t \geq 0, \\
u(x, 0)=0, \quad x \geq 0,
\end{gathered}
$$


and where it is assumed that $u(x, t)$ is bounded for large $x$. This may be considered a forward problem and is well posed. It has solution

$$
u(x, t)=\int_{0}^{t} \gamma(x, t-\tau) \psi(\tau) d \tau,
$$

where $\gamma(x, t)$ is the fundamental solution of the heat equation, namely,

$$
\gamma(x, t)=\frac{1}{\sqrt{\pi t}} e^{-x^{2} / 4 t} .
$$

Suppose, instead, that we solve an inverse problem by specifying

$$
u(1, t)=F(t)
$$

and that we wish to solve for $\psi(t)$. Then $\psi$ is a solution of the Volterra integral equation of the first kind

$$
F(t)=\int_{0}^{t} \gamma(1, t-\tau) \psi(\tau) d \tau
$$

well known to be ill posed.

We proceed as follows. We will suppose that $u(x, t)$ has a Laguerre expansion

$$
u(x, t)=\sum_{n=0}^{\infty} c_{n}(x) L_{n}(t) .
$$

The fundamental solution has a Laguerre expansion given in terms of the theta polynomials by (6.20). Denote $c_{n}(1)=a_{n}$ and $-c_{n}{ }^{\prime}(0)=b_{n}$. Then $a_{n}$ may be assumed known and we must solve for $b_{n}$ from

$$
\begin{aligned}
\sum_{n=0}^{\infty} a_{n} L_{n}(t)= & \int_{0}^{t}\left(\sum_{n=0}^{\infty} \frac{p_{n}^{(-1)}(1)}{e n !} L_{n}(t-\tau)\right)\left(\sum_{m=0}^{\infty} b_{m} L_{m}(\tau)\right) d \tau \\
= & \sum_{n=0}^{\infty} \sum_{m=0}^{\infty} \frac{p_{n}^{(-1)}(1)}{e n !} b_{m}\left(L_{m+n}(t)-L_{m+n+1}(t)\right) \\
= & \frac{p_{0}^{(-1)}(1) b_{0}}{e} L_{0} \\
& +\sum_{n=1}^{\infty}\left\{\frac{p_{0}^{(-1)}(1) b_{n}}{e}+\sum_{j=1}^{n}\left[\frac{p_{j}^{(-1)}(1)}{e j !}-\frac{p_{j-1}^{(-1)}(1)}{e(j-1) !}\right] b_{n-j}\right\} L_{n} .
\end{aligned}
$$

Hence, we have the recurrence

$$
\begin{gathered}
b_{0}=\frac{a_{0} e}{p_{0}^{(-1)}(1)} \\
b_{n}=\frac{1}{p_{0}^{(-1)}(1)}\left\{e a_{n}-\sum_{j=1}^{n}\left[\frac{p_{j}^{(-1)}(1)}{j !}-\frac{p_{j-1}^{(-1)}(1)}{(j-1) !}\right] b_{n-j}\right\} .
\end{gathered}
$$


The recurrence (7.12) is readily solved by generating functions. Multiplying both sides of (7.12) by $t^{n}$ and summing, we find

$$
\sum_{n=0}^{\infty} b_{n} t^{n}=\frac{e^{1 / \sqrt{1-t}}}{\sqrt{1-t}} \sum_{n=0}^{\infty} a_{n} t^{n} .
$$

It follows from (5.8) that the $b_{n}$ are given by the convolution sum

$$
b_{n}=e \sum_{k=0}^{n} \frac{p_{k}^{(-1)}(-1)}{k !} a_{n-k},
$$

which completely solves the inverse problem.

Both of the above applications are amenable to a regularization process and the second application may be readily adapted to multidimensional problems. These considerations will be addressed in future work.

This provides a fitting conclusion to our investigation of the theta functions, whose origins were a Laguerre series solution of the Cauchy problem of inverse heat conduction. It is deeply satisfying that the new polynomials should emerge from such a practical problem, and that the operational calculus should provide "a local habitation and a name."

Acknowledgments. The author thanks Dr. M. Pettigrew for bringing to his attention the work of Professor Rota and colleagues. The suggestions of the referee were greatly appreciated.

\section{REFERENCES}

[1] J. Avery, Hyperspherical Harmonics and Generalized Sturmians, Progress in Theoretical Chemistry and Physics, vol. 4, Kluwer Academic Publishers, Dordrecht, 2000.

[2] L. Carlitz, A note on the Bessel polynomials, Duke Math. J. 24 (1957), 151-162.

[3] A. Di Bucchianico, Representations of Sheffer polynomials, Stud. Appl. Math. 93 (1994), no. 1, 1-14.

[4] A. Di Bucchianico, D. E. Loeb, and G.-C. Rota, Umbral calculus in Hilbert space, Mathematical Essays in Honor of Gian-Carlo Rota (Cambridge, Mass, 1996), Progr. Math., vol. 161, Birkhäuser Boston, Massachusetts, 1998, pp. 213238.

[5] H. W. Gould, Combinatorial Identities, Henry W. Gould, West Virginia, 1972.

[6] M. Greguš, Third Order Linear Differential Equations, Mathematics and Its Applications (East European Series), vol. 22, D. Reidel Publishing, Dordrecht, 1987.

[7] C. Lanczos, Applied Analysis, Dover Books on Advanced Mathematics, Dover Publications, New York, 1988.

[8] N. N. Lebedev, Special Functions and Their Applications, Dover Publications, New York, 1972.

[9] D. Loeb and G.-C. Rota, Recent contributions to the calculus of finite differences: a survey, Geometry and Complex Variables (Bologna, 1988/1990), Lecture Notes in Pure and Appl. Math., vol. 132, Dekker, New York, 1991, pp. 239276. 
[10] R. Mullin and G.-C. Rota, On the foundations of combinatorial theory. III. Theory of binomial enumeration, Graph Theory and Its Applications (Proc. Advanced Sem., Math. Research Center, Univ. of Wisconsin, Madison, Wis, 1969) (B. Harris, ed.), Academic Press, New York, 1970, pp. 167-213.

[11] M. F. Pettigrew and D. G. Meredith, A Laguerre expansion of the Cauchy problem for the diffusion equation, Inverse Problems 10 (1994), no. 5, 1165-1183.

[12] G. E. Roberts and H. Kaufman, Table of Laplace Transforms, W. B. Saunders, Pennsylvania, 1966.

[13] G.-C. Rota, D. Kahaner, and A. Odlyzko, On the foundations of combinatorial theory. VIII. Finite operator calculus, J. Math. Anal. Appl. 42 (1973), 684760.

[14] G.-C. Rota and B. D. Taylor, The classical umbral calculus, SIAM J. Math. Anal. 25 (1994), no. 2, 694-711.

[15] M. Rotenberg, Theory and application of Sturmian functions, Adv. At. Mol. Phys. 6 (1970), 233-268.

[16] G. Sansone, Studi sulle equazioni differenziali lineari omogenee di terzo ordine nel campo reale, Univ. Nac. Tucumán Rev. Ser. A 6 (1948), 195-253 (Italian).

[17] B. van der Pol and H. Bremmer, Operational Calculus Based on the Two-Sided Laplace Integral, Cambridge University Press, Cambridge, 1955.

D. G. Meredith: Department of Mathematics, King's College at the University of Western Ontario, 266 Epworth Avenue, London, Ontario, Canada N6A 2M3

E-mail address: meredi th@uwo.ca 


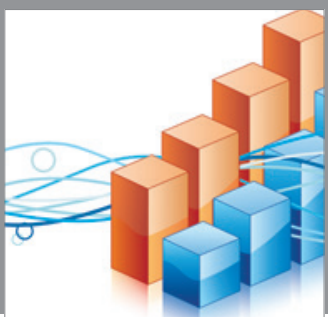

Advances in

Operations Research

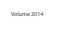

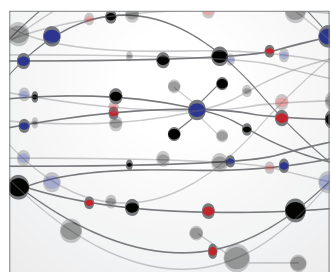

\section{The Scientific} World Journal
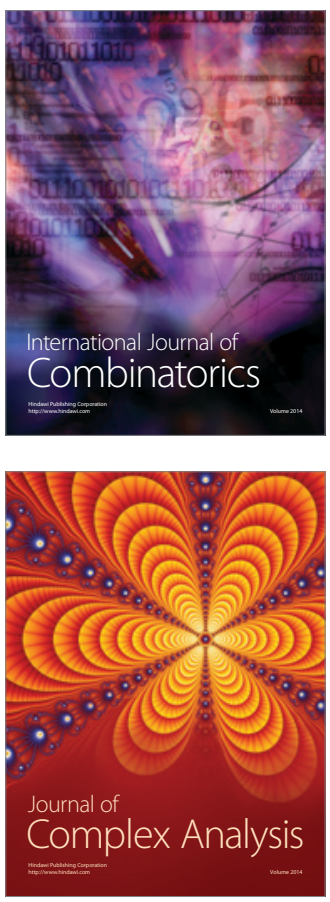

International Journal of

Mathematics and

Mathematical

Sciences
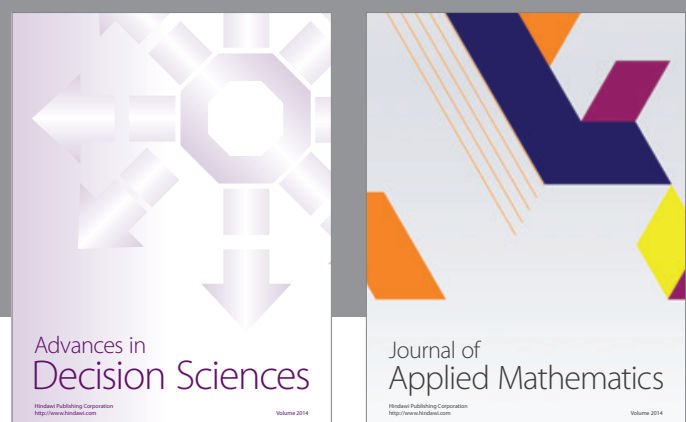

Journal of

Applied Mathematics
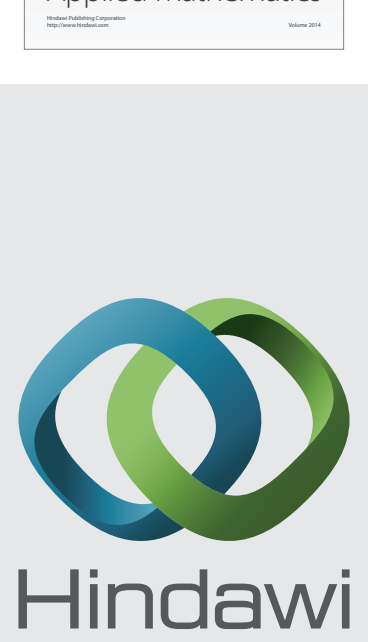

Submit your manuscripts at http://www.hindawi.com
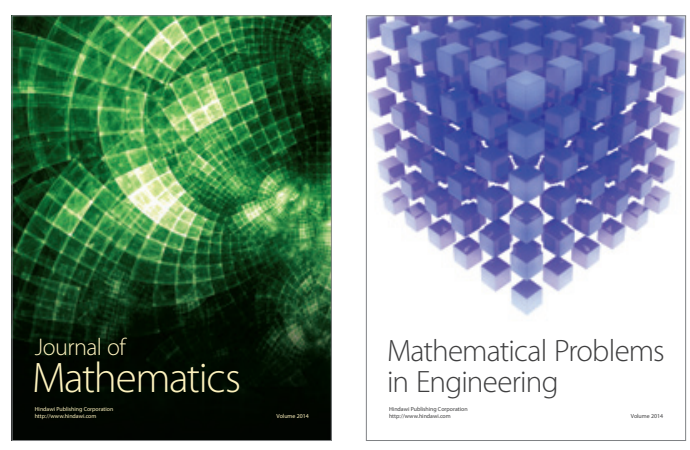

Mathematical Problems in Engineering
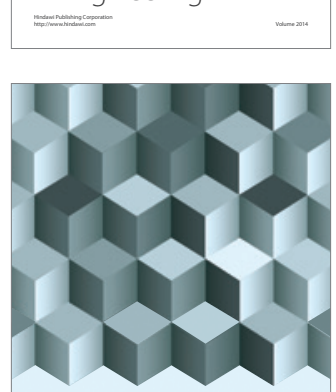

Journal of

Function Spaces
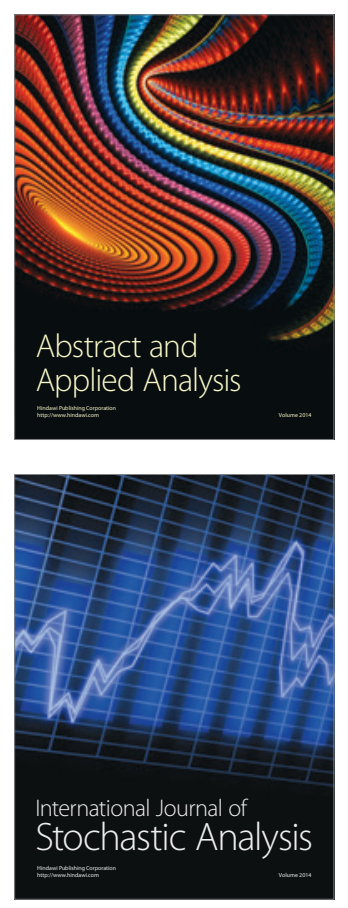

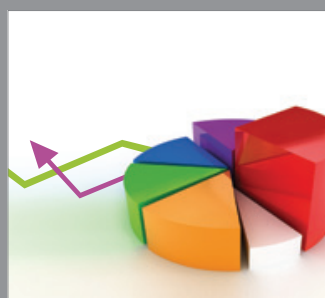

ournal of

Probability and Statistics

Promensencen
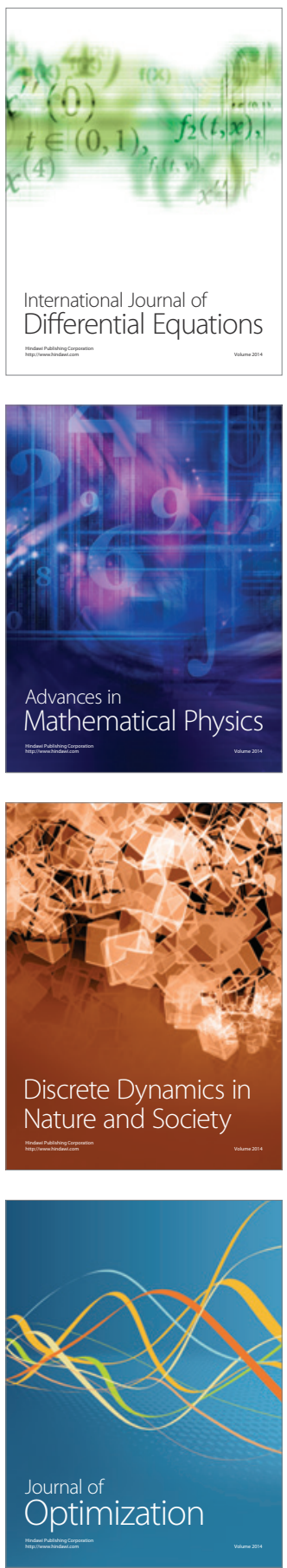